\title{
Function and mechanism of pyrin and IL-10 in the regulation of the inflammasome in pulmonary vascular endothelial cells following hemorrhagic shock
}

\author{
XIN JIN $^{1,2}$, YONGXING YAO ${ }^{1}$, XING LU $^{2}$, PENG XU ${ }^{2}$, YANFEI XIA ${ }^{2}$ and SHENGMEI ZHU ${ }^{1}$ \\ ${ }^{1}$ Department of Anesthesia, First Affiliated Hospital, Zhejiang University School of Medicine, Hangzhou, \\ Zhejiang 310003; ${ }^{2}$ Department of Anesthesia, Zhejiang Hospital, Hangzhou, Zhejiang 310013, P.R. China
}

Received May 7, 2018; Accepted May 23, 2019

DOI: $10.3892 /$ etm.2019.7757

\begin{abstract}
The present study aimed to evaluate the function of pyrin and interleukin-10 (IL-10) and the potential mechanisms underlying the regulation of inflammation in pulmonary vascular endothelial cells (ECs) following hemorrhagic shock (HS). Adult female Sprague-Dawley rats were divided into 4 groups ( $n=6$ in each group) to examine the changes in pyrin expression following HS-lipopolysaccharide (LPS) administration, including the following groups: A sham operation $(\mathrm{SM})+$ tracheal injection of saline (SAL) group; a HS + SAL group; a SM + LPS group (with a tracheal injection of endotoxin); and a HS + LPS group. An additional 4 groups were used to evaluate the function of IL-10, by the additional intratracheal injection of recombinant IL-10. Western blot analysis and immunofluorescence were performed in order to investigate the changes to pyrin and IL-10 expression in pulmonary vascular ECs. The expression levels of pyrin in the SM + LPS group were significantly increased in comparison with the $\mathrm{SM}+\mathrm{SAL}$ group $(\mathrm{P}<0.01)$. Additionally, the expression levels of pyrin were significantly increased in the HS + LPS group compared with the HS + SAL group $(\mathrm{P}<0.01)$. The expression levels of caspase-1 were significantly increased in the HS + LPS group compared with those in the other three groups $(\mathrm{P}<0.01)$. The expression levels of pyrin in the HS + LPS + IL-10 group were significantly increased compared with the HS + LPS group $(\mathrm{P}<0.01)$. The expression levels of caspase-1 were significantly decreased following
\end{abstract}

Correspondence to: Professor Yanfei Xia, Department of Anesthesia, Zhejiang Hospital, 12 Lingyin Road, Hangzhou, Zhejiang 310013, P.R. China

E-mail: xiayf0303@126.com

Dr Shengmei Zhu, Department of Anesthesia, First Affiliated Hospital, Zhejiang University School of Medicine, 79 Qingchun Road, Hangzhou, Zhejiang 310003, P.R. China

E-mail: smzhu20088@zju.edu.cn

Key words: hemorrhagic shock, pulmonary vascular endothelial cells, interleukin-10, pyrin
IL-10 treatment compared with those in the HS + LPS group $(\mathrm{P}<0.01)$. Therefore, HS attenuated LPS-induced pyrin expression in pulmonary vascular ECs and may also inhibit the expression of IL-10, resulting in the activation of caspase-1 subsequent to a second LPS insult.

\section{Introduction}

Hemorrhagic shock (HS) and subsequent resuscitation result in an overly active systemic immuno-inflammatory response that may produce an exaggerated physiological response, resulting in systemic inflammatory response syndrome (SIRS). Complications of SIRS often involve organ dysfunction and organ failure, including acute lung injury (ALI) and multiple organ dysfunction syndrome (MODS), with high mortality rates, ranging from $27-100 \%(1,2)$. Although substantial progress has been made in the treatment of HS, the incidence of SIRS following HS remains up to $43 \%$ (3), and there is no effective measure to prevent the patients from entering the SIRS-MODS stage.

The 'second hit' model proposed by Moore et al (4) is considered to be a good standard as an empirical explanation for HS-associated inflammation. HS, the first 'hit' primes the inflammatory system and a second 'hit', for example through infection, aggravates the already sensitized immune system (4). Inflammation is governed by interactions between proinflammatory and counter-inflammatory states; the imbalance between pro-inflammatory and anti-inflammatory responses serves a key role in the development of ALI and MODS (5), but the exact mechanism of HS sensitization to ALI and SIRS remains unclear. Therefore, it is of vital importance to investigate the mechanisms involved in the inflammatory response state following HS.

Evidence has revealed that the activation of pulmonary vascular endothelial cells (ECs) and EC-mediated inflammation serve important functions in organ injury following a hemorrhage (6). The pyrin protein, consisting of 781 amino acid residues, is expressed in neutrophils, monocytes and macrophages, and its expression is regulated by a variety of cytokines (7). The pyrin protein is able to inhibit the activation of caspase- 1 and inhibit the mature release of interleukin (IL)-1 $\beta$, a proinflammatory mediator (8) that is involved 
in lung inflammation (9). Caspase-1 is also synthesized as an inactive $45 \mathrm{kDa}$ protein (procaspase-1) that undergoes autocatalytic processing following assembly of the inflammasome in response to an appropriate stimulus $(10,11)$. Of the cytokines that are able to regulate the expression of pyrin, interleukin (IL)-10 is able to induce the expression of pyrin protein in macrophages (8). It has been demonstrated that the immunosuppressive cytokine IL-10 may inhibit the activation of monocytes/macrophages and decrease the release of cytokines and chemokines induced by lipopolysaccharides (LPS) (9). In vivo experiments of ischemia/reperfusion have revealed that IL-10 is able to effectively decrease structural organ damage; however, when a IL-10 neutralizing antibody or IL-10 knockout is used, the inflammatory reaction is aggravated (12).

Taking into consideration these diverse results, it was hypothesized that pyrin and IL-10 are involved in the regulation of inflammation in pulmonary vascular ECs, and that the decrease in IL-10 expression contributes to a severe inflammatory response.

\section{Materials and methods}

Animals. Adult female Sprague-Dawley rats ( $\mathrm{n}=100$; weight, 200-240 g; Zhejiang Laboratory Animal Center) were provided by the Experimental Animal Center of the First Affiliated Hospital, Zhejiang University School of Medicine (Zhejiang, China). The rats were maintained in a standard cage, in a $22-24^{\circ} \mathrm{C}$ environment, with a $12: 12 \mathrm{~h}$ light:dark cycle and ad libitum access to food and water. All experiments were performed in accordance with the institutional guidelines for animal care and welfare. The present study was approved by the Medical Ethics Committee of Zheijang Hospital.

Experimental protocol. Prior to the initiation of the experiment, the rats were fasted for $24 \mathrm{~h}$ with ad libitum access to water $(13,14)$, in order to avoid backflow and aspiration when the trachea was intubated, and the greatest weight loss observed was $16 \mathrm{~g}$ ( $\sim 7 \%$ of body weight). Following the induction of anesthesia with an intraperitoneal injection of $2 \%$ sodium pentobarbital solution (50 mg/kg; Westang Biotechenology, Co., Ltd.), the left femoral artery was cannulated with an external infusion device to allow for the monitoring of mean arterial pressure, blood sampling and resuscitation. HS was initiated by the withdrawal of arterial blood and the mean arterial pressure reached $40 \pm 5 \mathrm{mmHg}$ within $20 \mathrm{~min}$. Blood was collected into a $10 \mathrm{ml}$ heparinized syringe to prevent the blood from clotting. In order to exclude the effect of heparin on the experimental results, the control group was administered equal amounts of heparin through arterial catheterization. Subsequent to a hypotensive period of $1 \mathrm{~h}$, autologous blood transfusion was performed and the same quantity of lactate solution was administered (15). Resuscitation was completed within $20 \mathrm{~min}$. Following resuscitation, the catheters were removed and the artery was ligated to close the incision. The sham surgery (SM) group underwent femoral artery catheterization without blood loss. Following resuscitation for $2 \mathrm{~h}$, the rats were administered LPS (cat. no., L2880; Sigma-Aldrich; Merck KGaA) at a dose of $100 \mu \mathrm{g} / \mathrm{kg}$ body weight $(16,17)$, which was intratracheally injected as the second 'hit' model of HS-LPS. During the entire experiment, all rats were under anesthesia.

The experiment was divided into two parts. The first part was used to confirm that pulmonary ECs participated in the inflammatory process subsequent to the second 'hit' and confirm whether the LPS-mediated upregulation of pyrin expression was impaired following HS. The rats were divided into 4 groups ( $n=6$ for each group), as follows: i) A $\mathrm{SM}+$ tracheal injection of saline (SAL) group; this was the negative control group; ii) a HS + SAL group; iii) a SM + LPS group. with a tracheal injection of endotoxin; and iv) a HS + LPS group. A total of $8 \mathrm{~h}$ after tracheal injection, the rats were euthanized by administering an overdose of sodium pentobarbital solution $(200 \mathrm{mg} / \mathrm{kg})$ followed by cervical dislocation. Alveolar lavage was performed, lung tissues were collected and stored in liquid nitrogen, and then western blot analysis and immunofluorescence were performed.

The second part was performed to clarify the potential mechanism of the inhibition of the LPS-mediated upregulation of pyrin expression following HS. Rats were divided into four groups, as follows: i) A SM + LPS group; ii) a HS + LPS group (negative control group); ii) a HS + LPS + intratracheal injection of saline (NS) group; and iv) a HS + LPS + IL-10, with an intratracheal injection of recombinant IL-10 (BioLegend, Inc.) group. A total of $8 \mathrm{~h}$ after tracheal injection, the rats were euthanized by administering an overdose of sodium pentobarbital solution $(200 \mathrm{mg} / \mathrm{kg})$ followed by cervical dislocation. Alveolar lavage was performed, lung tissues were collected and stored in liquid nitrogen, and then western blot analysis was performed.

Immunofluorescence. The lung tissues were fixed in $4 \%$ paraformaldehyde at $4{ }^{\circ} \mathrm{C}$ overnight. The lung tissue of each group was serially sliced into $4-\mu \mathrm{m}$ thick slices, and then incubated with $5 \%$ goat serum (Beyotime Institute of Biotechnology) at $22^{\circ} \mathrm{C}$ for $1 \mathrm{~h}$. Lung tissues from each group were sliced into 2 sections, and each sample was incubated for $48 \mathrm{~h}$ at $4^{\circ} \mathrm{C}$ with cluster of differentiation (CD)34 mouse primary antibodies (cat. no., 60108-1-lg) and anti-pyrin rabbit primary antibodies (cat. no., 24280-1-AP; both 1:100; ProteinTech Group, Inc.) respectively. CD34, a cell surface sialomucin-like glycoprotein, is commonly used as a marker for identifying vascular ECs (18). Vascular ECs stained with CD34 exhibit a red color in the cytoplasm. PBS was used as blank control instead of the primary antibody. Subsequent to washing with PBS 3 times (3 min each), the slides were incubated with Cy3-conjugated goat anti-mouse immunoglobulin (Ig)G (cat. no., P0193) and FITC-conjugated goat anti-rabbit IgG (cat. no., P0186; both 1:100; Beyotime Institute of Biotechnology) for $1 \mathrm{~h}$ at room temperature. The staining method was performed according to the manufacturer's protocol of immunofluorescence kits (Immunol Fluorence Staining Kit with Cy3-Labeled Goat Anti-Mouse IgG and Immunol Fluorescence Staining Kit with FITC-Labeled Goat Anti-Rabbit IgG; both Beyotime Institute of Biotechnology). Images were obtained using a wide-field fluorescence microscope (Olympus X-cite 120; Olympus Corporation; magnification, x200).

Western blot analysis. Lung tissues of rats were homogenized on ice and lysed in lysis buffer which was prepared as follows: 


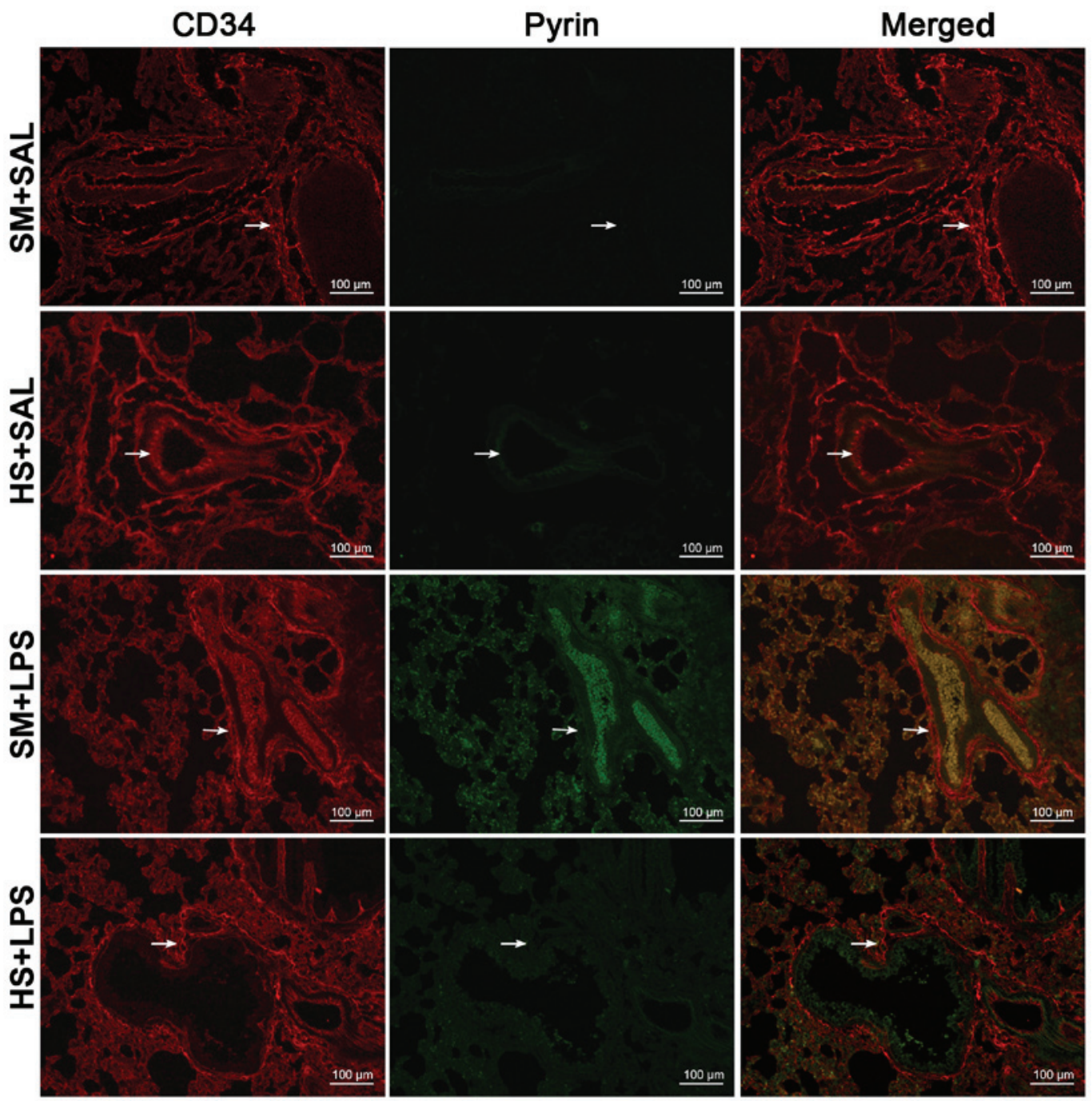

Figure 1. HS attenuates the LPS-induced pyrin expression in pulmonary vascular ECs. Immunofluorescence staining analysis was conducted. Pulmonary vascular endothelial cells (white arrows) were incubated with anti-CD34 and anti-pyrin antibodies. The CD34-positive cells (red) presented a typical cobblestone-like morphology. The merged images (yellow) demonstrate overlap of pyrin (green) and CD34 (red). Magnfication, x200. SM, sham operation; SAL, tracheal injection of saline; HS, hemorrhagic shock; LPS, tracheal injection of lipopolysaccharide. CD34, cluster of differentiation 34.

$10 \mathrm{mM}$ Tris (pH 7.4), $5 \mathrm{mM}$ EDTA, $150 \mathrm{mM} \mathrm{NaCl}, 10 \mathrm{mM}$ NaF, $1 \%$ Triton X-100, $1 \mathrm{mM} \mathrm{Na}_{3} \mathrm{VO}_{4}, 20 \mathrm{mM} \mathrm{PMSF}, 10 \mu \mathrm{g} / \mathrm{ml}$ leupeptin and $10 \mu \mathrm{g} / \mathrm{ml}$ aprotinin. The tissue homogenate was centrifuged at $1,000 \mathrm{x} \mathrm{g}$ in an Eppendorf centrifuge (Eppendorf) at $4^{\circ} \mathrm{C}$ for $10 \mathrm{~min}$, and then the protein content in the supernatants were quantified using the Bradford method. Proteins $(30 \mu \mathrm{g} /$ lane $)$ for each sample were separated using $10 \%$ SDS-PAGE gel and then transferred onto a polyvinylidene fluoride membrane. Subsequent to being blocked by $5 \%$ non-fat milk for $1 \mathrm{~h}$ at $22^{\circ} \mathrm{C}$ and then for $24 \mathrm{~h}$ at $4^{\circ} \mathrm{C}$, the membranes were incubated with the primary antibodies against pyrin (1:1,000; cat. no., 24280-1-AP; ProteinTech Group, Inc.) and caspase-1 (1:2,000; cat. no., ab188326; Abcam). The blots were then washed and incubated with horseradish peroxidase (HRP)-labeled goat anti-rabbit IgG (1:2,000; cat. no., A0208; Beyotime Institute of Biotechnology) and HRP-labeled goat anti-mouse IgG (1:2,000; cat. no., A0216; Beyotime Institute of Biotechnology) for $2 \mathrm{~h}$ at room temperature. Protein bands were then detected by enhanced chemiluminescence. A ChemiDoc MP System (Bio-Rad Laboratories, Inc., Hercules, CA, USA) was used to capture the protein bands and then
Image Lab 5.0 software (Bio-Rad Laboratories, Inc.) was used to analyze the bands. GAPDH and pro-caspase-1 were used as loading controls. Molecular weight standards were used from commercial markers (Thermo Fisher Scientific Inc.).

Statistical analysis. SPSS v20.0 software (IBM Corp.) was applied for statistical analysis and the experiments were performed and repeated three times. Data are presented as the mean \pm standard error of the mean. Data were analyzed using one-way analysis of variance followed by a Student-Neuman-Keuls post hoc test. $\mathrm{P}<0.05$ was considered to indicate a statistically significant difference.

\section{Results}

HS attenuates the LPS-induced pyrin expression in pulmonary vascular ECs. Subsequent to the removal of the majority of the macrophages following lung lavage, an analysis of the distribution of ECs and pyrin was performed. In immunofluorescence staining, cells stained with CD34 (red) were considered to be pulmonary vascular ECs. As presented in Fig. 1, these 
A
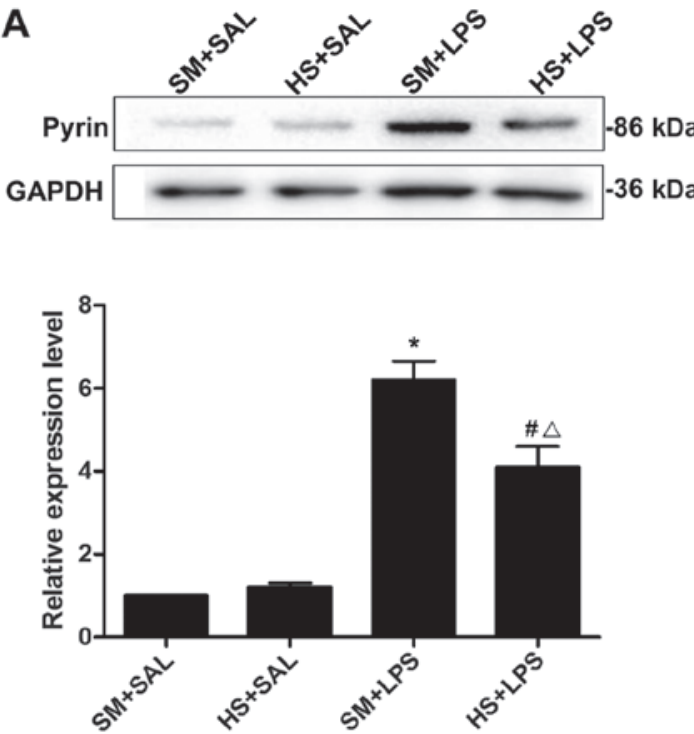

B
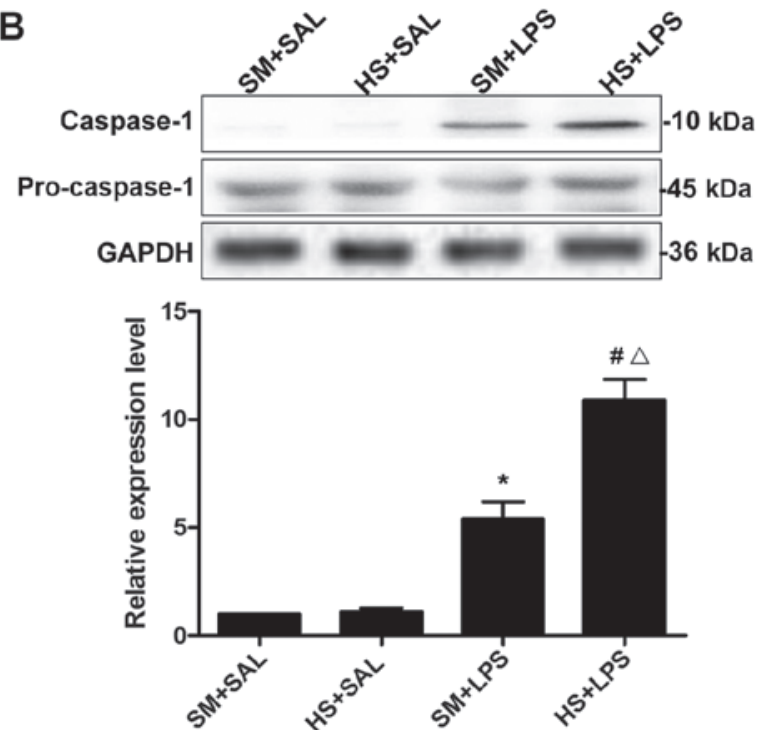

Figure 2. HS leads to a downregulation and upregulation LPS-induced pyrin and caspase-1 expression, respectively, in pulmonary vascular ECs. Western blot analysis was conducted. Expression changes of (A) pyrin and (B) caspase-1 following HS-LPS 'hit'. "P $<0.05$ vs. the SM $+\mathrm{SAL}$ group. "P<0.05 vs. the HS $+\mathrm{SAL}$ group. ${ }^{\Delta} \mathrm{P}<0.05$ vs. the SM + LPS group $(\mathrm{n}=6)$. SM, sham operation; SAL, tracheal injection of saline; HS, hemorrhagic shock; LPS, tracheal injection of lipopolysaccharide.

CD34-positive cells presented a typical cobblestone-like morphology. Pyrin staining (green) demonstrated the presence of pyrin in ECs. The second 'hit', HS-LPS, substantially decreased the expression of pyrin protein in pulmonary vascular ECs. Furthermore, the expression of pyrin was evaluated in the supernatant of the lung homogenate (Fig. 2A). No difference regarding the expression levels of pyrin between the SM + SAL group and the HS + SAL group was observed $(\mathrm{P}>0.05)$. The expression levels of pyrin in the SM + LPS group were significantly increased in comparison with that in the $\mathrm{SM}+\mathrm{SAL}$ group $(\mathrm{P}<0.01)$, demonstrating that LPS stimulation had significantly increased the pyrin protein expression level in pulmonary vascular ECs. Additionally, the expression of pyrin was significantly increased in the HS + LPS group compared with the HS + SAL group $(\mathrm{P}<0.01)$. However, the pyrin expression level in the HS + LPS group was significantly decreased compared with that of the SM + LPS group $(\mathrm{P}<0.01)$, indicating that HS weakened the expression of pyrin induced by LPS.

As presented in Fig. 2B, there was no significant difference with regard to the expression of caspase- 1 in the SM + SAL and $\mathrm{HS}+\mathrm{SAL}$ groups $(\mathrm{P}>0.05)$. The expression of caspase- 1 in the $\mathrm{SM}+\mathrm{LPS}$ was increased compared with that in the SM + SAL group $(\mathrm{P}<0.01)$, demonstrating that LPS stimulation increased the expression of caspase- 1 protein in pulmonary vascular ECs. In addition, the expression of caspase-1 in the HS + LPS group was significantly increased compared with that in the $\mathrm{SM}+$ LPS group $(\mathrm{P}<0.01)$. Collectively, HS augmented the LPS-induced caspase-1 expression.

Involvement of IL-10 in regulating pyrin expression changes in pulmonary vascular ECs following the second 'hit' of HS-LPS. The expression of the pyrin protein is regulated by various cytokines, including IL-10 (8). The present study assessed the hypothesis that HS damaged the expression of IL-10, thereby affecting the expression of the pyrin protein, which resulted in the activation of caspase- 1 subsequent to the second 'hit' of HS-LPS. As presented in Fig. 3A, the expression levels of pyrin in the SM + LPS group were significantly increased in comparison with the HS + LPS group $(\mathrm{P}<0.01)$; and the expression levels of pyrin in the HS + LPS + IL-10 group were significantly increased compared with the HS + LPS group $(\mathrm{P}<0.01)$. The results demonstrated that HS and the intratracheal injection of LPS may result in the expression of pyrin being significantly decreased, however, exogenous IL-10 treatment increases pyrin expression.

As presented in Fig. 3B, the expression levels of cleaved caspase-1 were significantly different between the SM + LPS and HS + LPS groups $(\mathrm{P}<0.01)$. The expression of caspase-1 was significantly decreased subsequent to IL-10 treatment compared with those in the HS + LPS group $(\mathrm{P}<0.01)$. Although HS promoted the LPS-induced caspase-1 expression, intratracheal IL-10 treatment may decrease the expression of caspase-1, alleviating the inflammatory reaction. Therefore, the IL-10 treatment-inhibited caspase- 1 activation induced by LPS may occur through the upregulation of pyrin expression in HS rats.

\section{Discussion}

Systemic inflammatory response and organ failure are the primary factors affecting mortality in patients with HS and trauma. How HS enhances the inflammatory response of the body remains unclear. The activation of caspase- 1 serves an important role in systemic inflammatory responses subsequent to shock (10). In the present study, it was revealed that LPS induced the activation of caspase- 1 and also induced the expression of pyrin protein in pulmonary vascular ECs. The increase of pyrin protein expression may inhibit the activation of caspase-1 and form a negative feedback pathway. Notably, the increased expression of IL-10 induced by LPS in lung ECs may enhance the expression levels of pyrin in lung ECs and enhance the negative feedback regulation of inflammation. However, HS, by inhibiting the expression of IL-10, weakens 
A
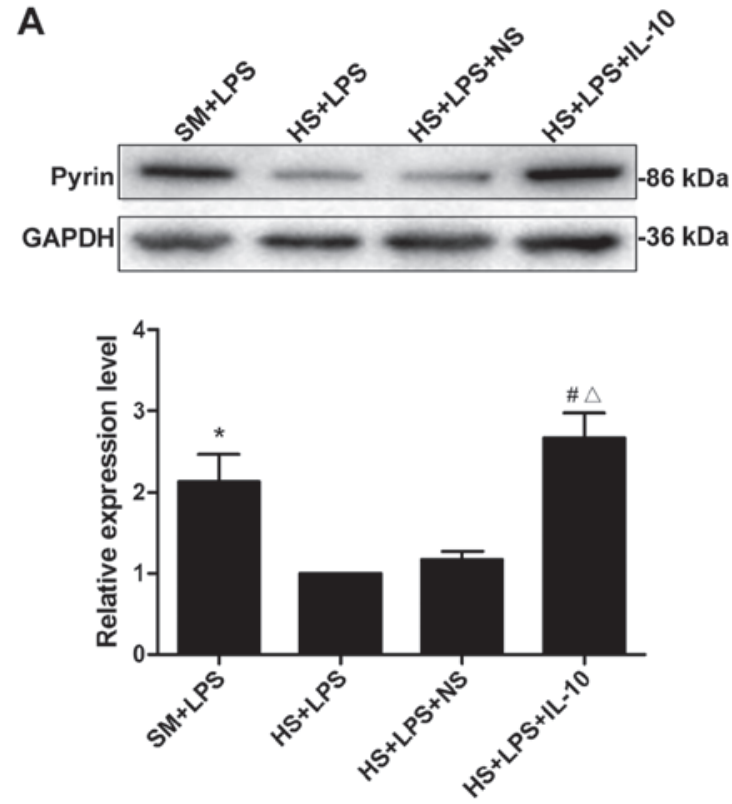

B
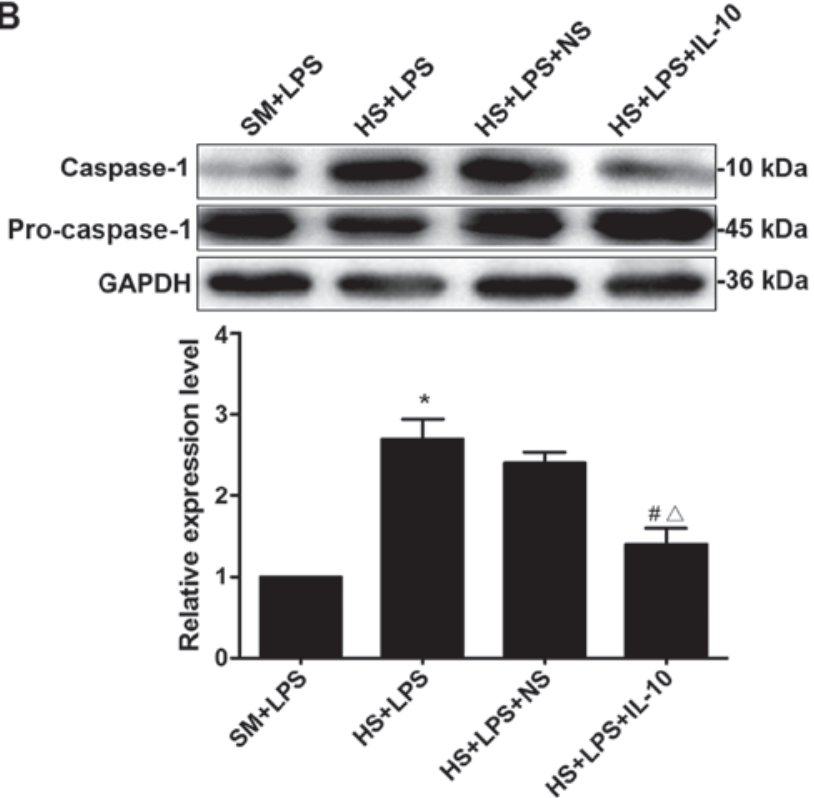

Figure 3. Involvement of IL-10 in regulating pyrin expression changes in pulmonary vascular ECs following the second 'hit' of HS-LPS. Western blot analysis was conducted. (A) Expression changes of pyrin following HS-LPS 'hit' and exogenous recombinant IL- 10 treatment. *P<0.05 vs. HS + LPS group. "P $<0.05$ vs. HS + LPS group. ${ }^{\Delta} \mathrm{P}<0.05$ vs. HS + LPS + NS group (n=6). (B) Expression changes of caspase-1 following HS-LPS 'hit' and exogenous recombinant IL-10 treatment. ${ }^{*} \mathrm{P}<0.05$ vs. SM + LPS group. ${ }^{\#} \mathrm{P}<0.05$ vs. HS + LPS group. ${ }^{\Delta} \mathrm{P}<0.05$ vs. HS + LPS + NS group (n=6). SM, sham operation; NS, intratracheal injection of saline; HS, hemorrhagic shock; LPS, tracheal injection of lipopolysaccharide; IL-10, interleukin-10.

the expression of pyrin, thereby damaging the negative feedback regulation of the inflammatory response and resulting in the enhancement of inflammatory body activation and consequently increasing the release of caspase-1.

The pyrin protein may inhibit the activation of caspase-1, and subsequently inhibit the maturation of inflammatory bodies. The destruction of the $\mathrm{C}$ terminal of the pyrin protein may enhance the sensitivity of mice to endotoxins and increase the activation of caspase-1, which indicates that the pyrin protein serves an important role in the maturation of inflammatory bodies (19). The pyrin protein interacts with caspase-1, apoptosis-associated speck-like protein containing a CARD, NLR family pyrin domain containing (NLRP)1, NLRP2, NLRP3 and IL-1B (precursors of inflammatory components) through its SPIa/ryanodine receptor and pyrin structural domains, thereby functioning as an inhibitor of the inflammatory body (20). A previous study has demonstrated that pyrin mutations may result in the activation of the NLRP3 independent inflammatory body, causing autoimmune diseases (21). The present study suggested that pyrin may result in different inflammatory outcomes through multiple different pathways.

IL-10 serves an important role in limiting inflammation and maintaining immune homeostasis (22). In acute respiratory distress syndrome, IL-10 has been demonstrated to inhibit proinflammatory mediators produced by pulmonary macrophages (23). In a previous clinical study, patients with acute respiratory distress syndrome, but with low levels of circulating and alveolar lavage fluid IL-10 exhibited an increased mortality rate compared with those with high IL-10 levels (24). At the gene level, a previous study indicated that, compared with pure LPS stimulation, hemorrhagic shock combined with LPS may significantly decrease the transcription of IL-10 mRNA (25). Patients that succumbed to mortality due to sepsis exhibited a decrease in IL-10 secretion (26), indicating that exogenous IL-10 had protective effects in sepsis or acute pancreatitis models $(26,27)$. A limitation of the present study is the absence of an in vitro model. A previous study confirmed that an in vitro HS model inhibited the LPS-induced increase of IL-10 expression at the gene level, which is closely associated with the progression of HS-induced pulmonary inflammation (28). It has been suggested that pyrin may serve an inhibitory role in casepase-1 activation and inhibit the development of inflammation $(29,30)$. In the present study, it was revealed that IL-10 may inhibit the activation of inflammatory cells by inducing the expression of pyrin in pulmonary vascular ECs. It was also confirmed that LPS induced the IL-10-based upregulated pyrin expression in lung ECs. The LPS treatment that induced pyrin protein expression in pulmonary vascular ECs was weakened by HS and LPS in the second 'hit' model. However, exogenous IL-10 may reverse the inhibition of pyrin expression in HS. It was additionally confirmed that in pulmonary vascular ECs, IL-10 was able to inhibit HS in a rat model of LPS-induced caspase-1 activation in the lungs, and the data from the present study revealed that in pulmonary vascular ECs, the expression of IL-10 may induce pyrin, while decreasing the activation of the inflammasome, thereby serving an important role in lung injury that occurs subsequent to HS. Furthermore, the present study had certain limitations: In addition to those aforementioned, only exogenous IL-10 was used, and the results obtained were used to examine our hypothesis. Due to the lack of measurement of the other endogenous dynamic changes following $\mathrm{HS}$ in this experiment, the association between pyrin and endogenous IL-10 requires additional verification.

In conclusion, it was proposed that HS attenuated the LPS-induced pyrin expression in pulmonary vascular ECs and that HS may also damage the expression of IL-10, thereby 
decreasing the expression of the pyrin protein in pulmonary vascular ECs, resulting in the activation of caspase-1 following the second LPS 'hit'. Additional studies investigating the expression changes and mechanism of pyrin in pulmonary vascular ECs are required to identify the underlying mechanism of SIRS induced by HS and novel targets for clinical intervention.

\section{Acknowledgements}

Not applicable.

\section{Funding}

The present study was supported by the Zhejiang Provincial Natural Science Foundation of China (grant no.,LY15H150005) and Medical Health Science and Technology Project of Zhejiang Provincial Health Commission (grant no., 2017KY176).

\section{Availability of data and materials}

The datasets used and/or analyzed during the current study are available from the corresponding author on reasonable request.

\section{Authors' contributions}

$\mathrm{XJ}$ and YY performed the majority of the experiments. XJ, YY and XL wrote the draft manuscript. XL also participated in the experimental design and helped aquire and analyze the experimental data. YY and PX designed and planned the implementation of the study. YX and SZ revised the manuscript, and agree to be accountable for all aspects of the work in ensuring that questions related to the accuracy or integrity of any part of the work are appropriately investigated and resolved. All authors read and approved the final manuscript.

\section{Ethics approval and consent to participate}

The study was approved by the Medical Ethics Committee of Zhejiang Hospital.

\section{Patient consent for publication}

Not applicable.

\section{Competing interests}

The authors declare that they have no competing interests.

\section{References}

1. El-Menyar A, El-Thani A, El Rasheid Z, Ahmad Z, Tuma M, AbdulRahman H, Parchani A, Peralta R and Latifi R: Multiple organ dysfunction syndrome (MODS): Is it preventable or inevitable. Int J Clin Med 3: 722-730, 2012.

2. van Wessem KJP and Leenen LPH: Reduction in mortality rates of postinjury multiple organ dysfunction syndrome: A shifting paradigm? A prospective population-based cohort study Shock 49: 33-38, 2018.

3. Anne Morrison C, Moran A, Patel S, Vidaurre Mdel P Carrick MM and Tweardy DJ: Increased apoptosis of peripheral blood neutrophils is associated with reduced incidence of infection in trauma patients with hemorrhagic shock. J Infect 66 : 87-94, 2013.
4. Moore FA, Sauaia A, Moore EE, Haenel JB, Burch JM and Lezotte DC: Postinjury multiple organ failure: A bimodal phenomenon. J Trauma 40: 501-512, 1996.

5. Minei JP, Cuschieri J, Sperry J, Moore EE, West MA, Harbrecht BG, O'Keefe GE, Cohen MJ, Moldawer LL, Tompkins RG, et al: The changing pattern and implications of multiple organ failure after blunt injury with hemorrhagic shock. Crit Care Med 40: 1129-1135, 2012.

6. Li Y, Xiang M, Yuan Y, Xiao G, Zhang J, Jiang Y, Vodovotz Y, Billiar TR, Wilson MA and Fan J: Hemorrhagic shock augments lung endothelial cell activation: Role of temporal alterations of TLR4 and TLR2. Am J Physiol Regul Integr Comp Physiol 297: R1670-R1680, 2009.

7. Chae JJ, Wood G, Richard K, Jaffe H, Colburn NT, Masters SL, Gumucio DL, Shoham NG and Kastner DL: The familial Mediterranean fever protein, pyrin, is cleaved by caspase-1 and activates NF-kappaB through its N-terminal fragment. Blood 112: 1794-1803, 2008.

8. Chae JJ, Komarow HD, Cheng J, Wood G, Raben N, Liu PP and Kastner DL: Targeted disruption of pyrin, the FMF protein, causes heightened sensitivity to endotoxin and a defect in macrophage apoptosis. Mol Cell 11: 591-604, 2003.

9. He X, Qian Y, Li Z, Fan EK, Li Y, Wu L, Billiar TR, Wilson MA, Shi $X$ and Fan J: TLR4-upregulated IL-1 $\beta$ and IL-1RI promote alveolar macrophage pyroptosis and lung inflammation through an autocrine mechanism. Sci Rep 6: 31663, 2016.

10. Martinon F, Burns K and Tschopp J: The inflammasome: A molecular platform triggering activation of inflammatory caspases and processing of proIL-beta. Mol Cell 10: 417-426, 2002.

11. Suzuki T, Franchi L, Toma C, Ashida H, Ogawa M, Yoshikawa Y, Mimuro H, Inohara N, Sasakawa C and Nuñez G: Differential regulation of caspase-1 activation, pyroptosis, and autophagy via Ipaf and ASC in Shigella-infected macrophages. PLoS Pathog 3: e111, 2007.

12. Welborn BM III, Moldawer LL, Seeger JM, Minter RM and Huber TS: Role of endogenous interleukin-10 in local and distant organ injury after visceral ischemia-reperfusion. Shock 20: 35-40, 2003.

13. Molthen RC: A simple, inexpensive, and effective light-carrying laryngoscopic blade for orotracheal intubation of rats. J Am Assoc Lab Anim Sci 45: 88-93, 2006.

14. Zhu X, Hu H, Li Z, Lin R, Mao J and Chen L: Gua Lou Gui Zhi decoction attenuates post-stroke spasticity via the modulation of GABAB receptors. Mol Med Rep 12: 5957-5962, 2015.

15. Zhou R, Hu DY, Liu LM and Zhou XW: Protective effects of apocynin on 'two-hit' injury induced by hemorrhagic shock and lipopolysaccharide. Acta Pharmacol Sin 23: 1023-1028, 2002.

16. Ulich TR, Fann MJ, Patterson PH, Williams JH, Samal B, Del CJ, Yin S, Guo K and Renick DG: Intratracheal injection of LPS and cytokines. V. LPS induces expression of LIF and LIF inhibits acute inflammation. Am J Physiol 267: L442-L446, 1994.

17. Tawadros PS, Powers KA, Yang I, Becker DA, Ginsberg MD, Szaszi K, Kapus A and Rotstein OD: Stilbazulenyl nitrone decreases oxidative stress and reduces lung injury after hemorrhagic shock/resuscitation and LPS. Antioxid Redox Signal 9: 1971-1977, 2007.

18. Testa JE, Chrastina A, Oh P, Li Y, Witkiewicz H, Czarny M, Buss $T$ and Schnitzer JE: Immunotargeting and cloning of two CD34 variants exhibiting restricted expression in adult rat endothelia in vivo. Am J Physiol Lung Cell Mol Physiol 297: L251-L262, 2009.

19. Feldmeyer L, Keller M, Niklaus G, Hohl D, Werner S and Beer HD: The inflammasome mediates UVB-induced activation and secretion of interleukin-1beta by keratinocytes. Curr Biol 17: 1140-1145, 2007.

20. Martinon F, Hofmann $\mathrm{K}$ and Tschopp J: The pyrin domain: A possible member of the death domain-fold family implicated in apoptosis and inflammation. Curr Biol 11: R118-R120, 2001.

21. Chae J, Cho YH, Lee GS, Cheng J, Liu PP, Feigenbaum L, Katz SI and Kastner DL: Gain-of-function Pyrin mutations induce NLRP3 protein-independent interleukin-1 $\beta$ activation and severe autoinflammation in mice. Immunity 34: 755-768, 2011.

22. Iyer SS and Cheng G: Role of interleukin 10 transcriptional regulation in inflammation and autoimmune disease. Crit Rev Immunol 32: 23-63, 2012.

23. Lo C, Fu M and Cryer HG: Interleukin 10 inhibits alveolar macrophage production of inflammatory mediators involved in adult respiratory distress syndrome. J Surg Res 79: 179-184, 1998.

24. Armstrong L and Millar AB: Relative production of tumour necrosis factor alpha and interleukin 10 in adult respiratory distress syndrome. Thorax 52: 442-446, 1997. 
25. Khadaroo RG, Fan J, Powers KA, Fann B, Kapus A and Rotstein OD: Impaired induction of IL-10 expression in the lung following hemorrhagic shock. Shock 22: 333-339, 2004.

26. Yeh FL, Shen HD and Fang RH: Deficient transforming growth factor beta and interleukin-10 responses contribute to the septic death of burned patients. Burns 28: 631-637, 2002.

27. Kusske AM, Rongione AJ, Ashley SW, McFadden DW and Reber HA: Interleukin-10 prevents death in lethal necrotizing pancreatitis in mice. Surgery 120: 284-289, 1996.

28. Cruz CM, Rinna A, Forman HJ, Ventura AL, Persechini PM and Ojcius DM: ATP activates a reactive oxygen species-dependent oxidative stress response and secretion of proinflammatory cytokines in macrophages. J Biol Chem 282: 2871-2879, 2007.
29. Halle A, Hornung V, Petzold GC, Stewart CR, Monks BG, Reinheckel T, Fitzgerald KA, Latz E, Moore KJ and Golenbock DT: The NALP3 inflammasome is involved in the innate immune response to amyloid-beta. Nat Immunol 9: $857-865,2008$

30. Watanabe H, Gaide O, Pétrilli V, Martinon F, Contassot E, Roques S, Kummer JA, Tschopp J and French LE: Activation of the IL-1beta-processing inflammasome is involved in contact hypersensitivity. J Invest Dermatol 127: 1956-1963, 2007.

(i) $\ominus$ This work is licensed under a Creative Commons Attribution-NonCommercial-NoDerivatives 4.0 International (CC BY-NC-ND 4.0) License. 